\title{
Absolute Parameters of Young Stars: V Puppis
}

\author{
E. Budding ${ }^{1,2,3,4}$, T. Love ${ }^{1,4}$, M. G. Blackford ${ }^{4}$, T. Banks ${ }^{5,6}$, \\ M. J. Rhodes ${ }^{7}$ \\ 1 Visiting astronomer, University of Canterbury Mt John Observatory, University of Canterbury, Private Bag 4800, Christchurch 8140, NZ; \\ 2 Carter Observatory, Wellington 6012, NZ; \\ 3 School of Chemical \& Physical Sciences, Victoria University of Wellington, Wellington 6012, New Zealand. \\ 4 Variable Stars South, RASNZ; \\ 5 Nielsen, Data Science, 200 W Jackson Blvd 17, Chicago, IL 60606, USA. \\ 6 Physics \& Astronomy, Harper College, $1200 \mathrm{~W}$ Algonquin Rd, Palatine, IL 60067, USA \\ 7 Brigham Young University', Provo, Utah, USA.
}

2 February 2021

\begin{abstract}
New spectrometric data on V Pup are combined with satellite photometry (HIPPARCOS and recent TESS) to allow a revision of the absolute parameters with increased precision. We find: $M_{1}=14.0 \pm 0.5, M_{2}=7.3 \pm 0.3\left(\mathrm{M}_{\odot}\right) ; R_{1}=$ $5.48 \pm 0.18, R_{2}=4.59 \pm 0.15\left(\mathrm{R}_{\odot}\right) ; T_{1} 26000 \pm 1000, T_{2} 24000 \pm 1000(\mathrm{~K})$, age $5 \pm 1(\mathrm{Myr})$, photometric distance 320 \pm 10 (pc). The TESS photometry reveals low-amplitude ( $\sim 0.002 \mathrm{mag})$ variations of the $\beta$ Cep kind, consistent with the deduced evolutionary condition and age of the optical primary. This fact provides independent support to our understanding of the system as in a process of Case A type interactive evolution that can be compared with $\mu^{1}$ Sco. The $\sim 10 \mathrm{M}_{\odot}$ amount of matter shed by the over-luminous present secondary must have been mostly ejected from the system rather than transferred, thus taking angular momentum out of the orbit and keeping the pair in relative close proximity. New times of minima for V Pup have been studied and the results compared with previous analyses. The implied variation of period is consistent with the Case A evolutionary model, though we offer only a tentative sketch of the original arrangement of this massive system. We are not able to confirm the previously reported cyclical variations having a $5.47 \mathrm{yr}$ period with the new data, though a direct comparison between the HIPPARCOS and TESS photometry points to the presence of third light from a star that is cooler than those of the close binary, as mentioned in previous literature.
\end{abstract}

Key words: stars: binaries (including multiple) close — stars: early type — stars: variable $\beta$ Cep type — stars: individual V Pup

\section{INTRODUCTION}

Studies of massive young stars, forming part of a 'Southern Binaries Programme', were presented by members of our group in a number of previous papers. General background was given by Budding (2008) and in the summary of Idaczyk et al. (2013). For a recent example see Blackford et al. (2019).

Binary stars are still the main source of fundamental data on stellar masses and radii. Our access to such advanced facilities as the high-precision photometry from the TESS satellite (Section 2) and the high-resolution HERCULES spectrometer (Section 3) for this application enables continued progress in refining our knowledge of the properties of stars. Popper's (1980) contribution to this subject set accuracy limits of a few percent, that was refined to an estimated $\sim 2 \%$ for the 45 examples studied by Andersen (1991). These review papers report an ongoing development of our understanding of stellar astrophysics, on the basis of data that has been confirmed to be reliable and of high quality. Andersen et al. (1993) also drew attention to the interesting role of close young binaries in understanding the relationship of stellar properties to their galactic environment. As well, Rucinski (2006) pointed out the observational neglect of binaries with declinations south of declination $-15^{\circ}$. Such discussion underlies and provides a springboard for the work presented here.

In this paper, we re-examine the early type eclipsing binary V Pup. The system consists of at least two young stars in a very close orbit, apparently in a 'Case A' process of interactive binary evolution. V Pup has attracted recent attention regarding the possibility that it may contain a black hole companion associated with its known high energy and microwave emissions (Giacconi et al., 1974; Groote et al., 1978; Bahcall et al., 1975; Qian et al., 2008; Maccarone et al., 2009). The issue may be probed more fully with the aid 
of up-to-date measurement and data-analysis procedures as well as the observational facilities mentioned above.

\subsection{Puppis}

V Pup ( = HD 65818, HIP 38597, WDS J07582-4915A, HR 3129; Gaia DR2 55171716782683628800) is a bright $(V=$ 4.41; $B-V=-0.17 U-B=-0.96$; types B1Vp + B2) eclipsing variable of the ' $\mathrm{EB}$ ' type, discovered by Williams (1886). It is located on the sky about $2.5^{\circ}$ south-west of, and at a comparable distance $(\sim 300 \mathrm{pc})$ to, the massive multiple star $\gamma^{2}$ Vel. Early history of observations of the system is briefly reviewed in the paper of Cousins (1947).

There has been ongoing literature discussion about the absolute parameters of $\mathrm{V}$ Pup, with effects attributed to the presence of gas streams or mass transfer distorting radial velocity $(\mathrm{RV})$ data on the stellar components (the 'Struve-Sahade effect' — see e.g. Maury, 1920; Popper, 1947; Frieboes, 1962; York et al., 1976; Cester et al., 1977; Eaton, 1978; Koch et al., 1981, Andersen et al., 1983, Stickland et al., 1998). The spectral types were given as B1 V and B3 V by Freiboes (1962) with corresponding masses of about 17 and $9 \mathrm{M}_{\odot}$ (Popper, 1980). Andersen et al. (1983) revised the parametrization of the system with improved data and analysis procedures, dropping the dwarf luminosity (V) of the secondary, which could now be assigned a subgiant (IV) classification. Their masses were reduced somewhat from those used by Popper to 14.8 and $7.8 \mathrm{M}_{\odot}$, with error estimates of about $2 \%$ of these values. The orbital period is relatively short at 1.4545 d. Stickland et al. (1998) obtained noticeably lower masses from IUE spectrograms, though unfortunately, the coverage at one of the elongations was rather incomplete.

V Pup appears to have at least one physical companion, associated with the double star h4025, the other star being an 11 th mag object at a separation of about 6 arcsec. Two other fainter companions have also been linked to the massive earlytype close binary. York et al. (1976), using UV data from the Copernicus satellite, deduced the presence of an HII region around the binary with an angular extent of $\sim 3.5$ arcmin.

This hot young binary may have some physical resemblance to $\mu^{1}$ Sco (Budding et al., 2015) in showing a semi-detached, near-contact configuration (cf. Schneider et al., 1979; Andersen et al. 1983; Bell et al. 1987a, 1987b; Terrell et al. 2005), and with this type of hot massive binary this is often associated with Case A evolution, in which mass loss from the erstwhile primary continues while the star is still on the hydrogen-burning Main Sequence (Sybesma, 1986; Yakut \& Eggleton, 2005).

With its stable light curve and well defined eclipses, timings of the light minima of $\mathrm{V}$ Pup may allow the stability of the binary orbit to be checked by use of the ' $\mathrm{O}-\mathrm{C}$ ' (observed minus calculated) diagram. Periodic residuals in such data (Kreiner et al., 2000) have suggested the presence of a $\sim 10 \mathrm{M}_{\odot}$ unseen companion to the binary, with an orbital period of about 5 years (Qian, Liao \& Fernandez-Lajus, 2008). Absence of visible indications of such a massive component in the optical region lead to the idea that this component may be a black hole.

Qian et al. (2008) argued that the X-ray emission from V Pup is consistent with accretion of stellar winds from the hot binary towards such a black hole. Interestingly, its mass could then be determinable from the observed orbital effects, and independently of evolution scenarios for the overall system.

Triple systems with accreting components have been invoked in the past, for example, to provide a physically plausible scenario for the Cygnus X-1 system (Bahcall et al. 1975). Also, the occurrence of long additional periods in systems with accreting neutron stars and black holes (Priedhorsky \& Terrell, 1983; Gies \& Bolton 1984; Zdziarski et al. 2007) may be explained in this way. Longer timescale effects in the short period neutron-star binary $4 \mathrm{U} 2129+47$ were attributed to an observed F type wide companion (Garcia et al. 1989; Nowak, Heinz \& Begelman 2002), further substantiating such an hierarchical stellar system model. Maccarone et al. (2009) made observations aimed at testing the hypothesis that the $\mathrm{X}$-ray emission from V Pup is from accretion onto the putative black hole by searching for radio emission, since a high ratio of radio to X-ray flux should be a characteristic signature of accreting black holes of stellar mass. They invoked an X-ray image of the field using Chandra, thus confirming that the X-rays are indeed from V Puppis or its close $(\sim 0.5$ $\operatorname{arcsec})$ vicinity.

Maccarone et al. gave an upper limit on the radio flux of about $300 \mu \mathrm{Jy}$. V Pup was identified in its X-ray emission with a luminosity of about $3 \times 10^{31} \mathrm{erg} / \mathrm{sec}$. This value is much lower than what had been reported in low angular resolution surveys of the past. Maccarone et al.'s results were interpreted to show that the X-ray emission comes from mass transfer between the two B stars in the system. The possibility of X-ray emission from the black hole accreting stellar wind from one or both of the B stars could not be ruled out, however.

In fact, massive early type stars, such as those in the V Pup system, are known to produce strong winds driven by the radiation pressure from the high temperature stellar surfaces. In a binary system consisting of two such stars the winds will interact, producing exotic shock effects observed across the spectrum, but noticeably in the X-ray range (e.g. Pollock, 1987; Chlebowski \& Garmany, 1991; Corcoran, 1996) and as synchrotron radio-emission. Such emissions tend to have a complex, intermittent or highly variable behaviour, particularly in higher energy ranges, and observations do not always support the predictions of basic models (e.g. Prilutski \& Usov, 1976; Cherepashchuk, 1976; Stevens et al. 1992). As more data have been collected over the years, fuller recognition of these anomalies has grown and theory developed to introduce more refined models (Pittard, 2009; Parkin et al. 2011; Rauw \& Nazé, 2016). It seems likely that the high energy emissions noted for V Pup, as well as their variability, may be accounted for by such modelling, without requiring the more extreme configuration invoked by Qian et al. (2008).

In the next section, we present and analyse photometry of V Pup. We follow this with some new spectroscopic data in Section 3. Combination of the results from the photometric and spectroscopic analyses lead to our derived set of absolute parameters in Section 4, and we discuss $\mathrm{O}-\mathrm{C}$ analysis of the system in Section 5. These findings are discussed and interpreted in terms of relevant stellar astrophysical models in the Section 6. 

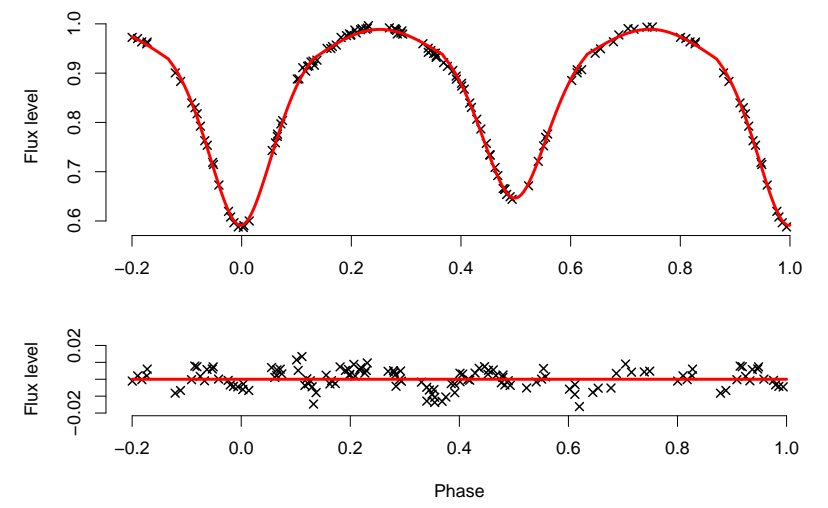

Figure 1. HIPPARCOS photometry of V Pup with Radau-model fitting. Residuals (crosses) are shown distributed about the lower axis.

\section{PHOTOMETRY}

As before in this programme we looked into previous photometry, particularly that of the HIPPARCOS satellite (ESA, 1997). The light-curve has been phased in the HIPPARCOS Epoch Photometry Annex according to the ephemeris Min I $=2448500.595+1.454507 \mathrm{E}$. Since the data were gathered over a $\sim 3$ year interval after JD 2447862.0708 a more appropriate epoch for the HIPPARCOS coverage would be HJD 2448455.5053; some 31 light cycles (45.090 d) earlier and close to the mean date of the data series. We adopted this for subsequent reference purposes.

A preliminary fitting to the HIPPARCOS light curve is shown in Fig 1 and a corresponding set of parameters listed in Table 1 . The mass ratio was fixed at 0.55 from initial radial velocity findings, discussed in the next section. Effective photospheric temperatures could be also estimated from the spectroscopy with the values shown in Table 1 . Formal error estimates are provided by the fitting program WiNFitTER that follow from the principles set out in Bevington (1969), chapter 11 . The procedure involves numerically inverting the determinacy Hessian of the $\chi^{2}$ variate in the vicinity of its minimum. This Hessian matrix should be positive definite for a properly posed inversion problem. The resulting errors then include the effects of inter-correlations between the parameters selected for adjustment (see also Banks \& Budding, 1990, for further background on this topic).

We can at once deduce, from the comparability of the luminosities despite the appreciable difference in masses, that we are not dealing with a Main Sequence pair. Kopal's (1959, Table 3.3) approximation that $r_{1}+r_{2} \approx 0.75$ for contact binaries, indicates that these stars must be relatively close to contact at the internal Lagrangian point $\mathrm{L}_{1}$. Indeed, the relative size of the secondary points to a filling of its 'Roche lobe'. This point, together with the closeness of the stars, confirms that this eclipsing binary is of the semi-detached Case-A-evolution type that may be compared with $\mu^{1}$ Sco (cf. Introduction).

A small shift from zero in the phase of minimum light $\Delta \phi_{0}$ is given in Table 1. The movement is in the sense that the observed minimum was late from its predicted time by an amount of some 0.0019 in phase, or 0.0028 days from the pre-
Table 1. Curve fitting results for archival HIPPARCOS photometry of V Pup using WinFitTer 6.2. Parameters for which no error estimate is given are adopted from information separate to the fitting. The HIPPARCOS raw data were phased according to the ephemeris provided in the HIPPARCOS Photometry Annex (ESA, 1997 ; - see text)

\begin{tabular}{lcc}
\hline & & \\
Parameter & Value & Error \\
\hline & & \\
$M_{2} / M_{1}$ & 0.55 & \\
$L_{1}$ & 0.58 & 0.01 \\
$L_{2}$ & 0.42 & 0.01 \\
$r_{1}(\operatorname{mean})$ & 0.406 & 0.002 \\
$r_{2}(\operatorname{mean})$ & 0.349 & 0.005 \\
$i($ deg $)$ & 75.1 & 0.4 \\
$T_{h}(\mathrm{~K})$ & 26000 & \\
$T_{c}(\mathrm{~K})$ & 24000 & \\
$u_{1}$ & 0.28 & \\
$u_{2}$ & 0.30 & \\
$\Delta \phi_{0}(\mathrm{deg})$ & 0.7 & 0.2 \\
$\chi^{2} / \nu$ & 1.02 & \\
$\Delta l$ & 0.006 & \\
\hline
\end{tabular}

dicted time using the HIPPARCOS ephemeris given above. We deduce that the time of observed minimum should be 24448455.5081 , or some 13618.01536 cycles after Kreiner's epoch that is used as a basis for $\mathrm{O}-\mathrm{C}$ studies. This leads to an $\mathrm{O}-\mathrm{C}$ value of $+0.0223 \mathrm{~d}$, which is slightly greater than the $+0.0198 \mathrm{~d}$ given by Qian et al. (2008).

\subsection{TESS photometry}

The Transiting Exoplanet Survey Satellite (TESS) was launched in April 2018, and began regular science operations by the end of July in that year. This NASA-supported mission is primarily aimed at a near complete survey of the whole sky, searching for exoplanet transiting systems (Ricker et al., 2015). In its first two years TESS should closely monitor over 200,000 Main Sequence stars with four wide-field optical CCD cameras. Photometry of the target stars is recorded with a 2 minute cadence. Additional images are taken of a wide ( 2200 sq. deg.) field every 30 minutes. It is planned to shorten the cadences to $20 \mathrm{sec}$ and 10 minutes in a later extension to the mission.

The data selected for the present study concerns about 15130 individual PDCSAP flux measures with the TESS identification of V Pup as tess2019006130736-s00070000000269562415-0131-s_lc. These data, from sector 7, run continuously in 2-minute samples from days BJD 2458492.628 to 2458516.088 , apart from a gap between 2458503.039 to 2458504.702. Additional TESS data on V Pup are available from sectors and 9, 34 and 35. The normalized PDCSAP fluxes, having a time-range of over 28 complete cycles of the binary system, were phased with the HIPPARCOS period and an adopted local epoch of BJD 2458492.98218. WINFITTER was then used to experiment with photometric modelling of these data.

Although the TESS data are of very high accuracy, enabling a remarkably close modelling fit (Fig 2), at an initial stage it became clear that there were small short-term com- 

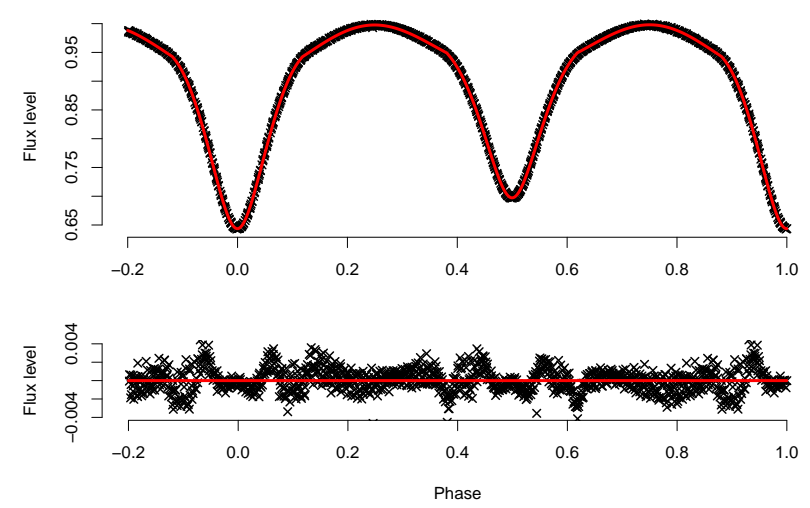

Figure 2. WinFitTER model fit to the complete TESS photometric data-set for V Pup, binned by a factor of 10 down to 1513 individual values. The scatter of individual readings is of order 0.0002 , but systematic departures of up to an order of magnitude greater than that can be seen in the residuals in the regions of the eclipses when plotted about the mean flux level 0.55 in this diagram. This is mostly accounted for by the fitting function's quadratic approximation being inadequate to represent the stars' photospheric limbdarkening effect with sufficient accuracy of detail. The parameters corresponding to this fitting are given in Table 2 .

plications in the form of the data compared with expectation for a standard binary system pattern of light variation. The complete data-set was then binned by a factor of 10 and an optimal model sought, using the results of Table 1 as a guide. The adopted model parameters are listed in Table 2 . The fitted light curve is shown in Fig 2, and Fig 3 displays a corresponding illustration of the basic stellar model.

These parameters have some noticeable differences from the ones in Table 1. But the forms of the light curves are also seen to be different: while both light curves show the peak brightness at close to unity and the minima are in about the same proportions, those in Fig 2 are more than $10 \%$ shallower than those of Fig 1. This is probably mainly due to the difference in effective wavelengths of the HIPPARCOS and TESS photometers. The TESS detector bandpass spans from $600-1000 \mathrm{~nm}$ and is centered on the Cousins I-band (7865 $\AA$ ), but with the high temperature expected for the source ( $25000 \mathrm{~K}$ - Table 2$)$, the effective wavelength works out at close to $731 \mathrm{~nm}$. On the other hand, the HIPPARCOS filter centres at $5593 \AA$ with a corresponding effective wavelength of 549 nm (Mann \& von Braun, 2015).

The possible contribution of an additional cooler component, or components, therefore arises (cf. Eaton, 1978). While there is no question that the TESS data have a higher measurement precision than those of HIPPARCOS, (by a couple of orders of magnitude, to judge by the scatter of individual measures), the TESS pixels (21 arcsec in extent) include significantly more background sky than the field of HIPPARCOS. The WDS (Worley \& Douglass, 1997) lists four visual companions to V Pup, but none of the nearest 3 within 20 arcsec are brighter than mag 11.5, while HJ 4025D, the brightest at $\mathrm{V}=9.88$ and separated at 40 arcsec, can not have added significantly to background illumination, though the possibility of error in the background subtraction procedure, given the large pixels of TESS, cannot be ruled out. Table 2

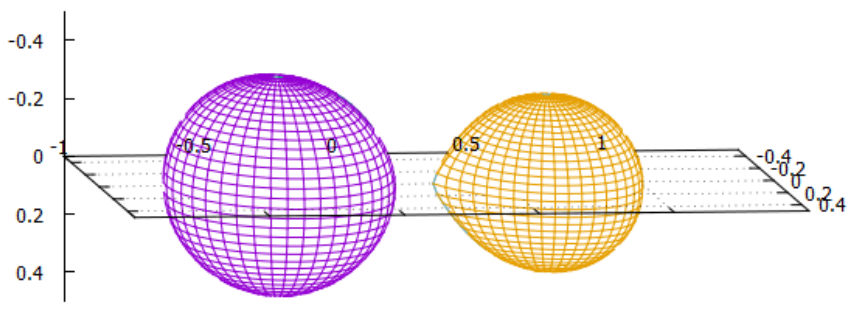

Figure 3. GNuplot illustration of V Pup using a standard Rochemodel corresponding to the parameters of Table 2 . The secondary is close to contact with its 'Roche lobe'.

presents this as $L_{3}$ : the additional light at the longer wavelength that reduces the apparent amplitude of the variation due to the close binary alone.

The residuals from the fitting to the binned complete dataset shown in Fig 2, whilst evidently less than those of Fig 1, reveal small systematic features, of amplitude $\lesssim 0.002$, that affect the eclipse phase ranges. These are associated mainly with shortcomings of the quadratic formula employed for the limb-darkening of the stars. Notwithstanding the eclipse regions, residuals in the out-of-eclipse phases are reasonably flat and close to zero.

When we examine individual light curves, however, we observe an additional intrinsic variation (see Figs 4 and 5) with a quasi-period of around 0.075 that of the system, i.e. $\approx 2.6 \mathrm{~h}$, that points to the massive B-type primary being a $\beta$ Cep type variable, with the rather low amplitude of $\sim 2$ mmag in the near IR region of the TESS filter. Anticipating the calibration of the primary's properties carried out in Section 4, we place that star in the H-R diagram in Fig 6, where indeed an incipient $\beta$ Cep variability is likely in view of the primary star's proximity to the instability strip. This discovery potentially offers an additional check on the system's parametrization.

Lomb-Scargle analysis (cf. Ruf, 1999) of the flux residuals reveals a complex pattern (Fig 7), with some 40 identified frequencies significant at the $99 \%$ confidence level. Nineteen of these are close to multiples of the orbital frequency, and therefore suspected to be aliases or artefacts from the close binary light curve modelling. In this connection, complications also arise from inadequacy of the limb-darkening series approximation. This is known to introduce additional oscillatory effects in the residuals from the eclipse phases. The PERIOd04 program (Lenz \& Breger 2005) was also used to check on the pulsations. Its pre-whitening technique was applied to data outside the two eclipses, from which some 13 significant frequencies were found, supporting the main clump of strong contributions in the $\sim P / 5-P / 10$ range. Further work is clearly needed to investigate and quantify the pulsation spectrum and its source.

\section{SPECTROSCOPIC DATA}

Spectroscopic data were mostly gathered using the High Efficiency and Resolution Canterbury University Large Échelle Spectrograph (HERCULES) of the Department of Physics and Astronomy, University of Canterbury, New Zealand 

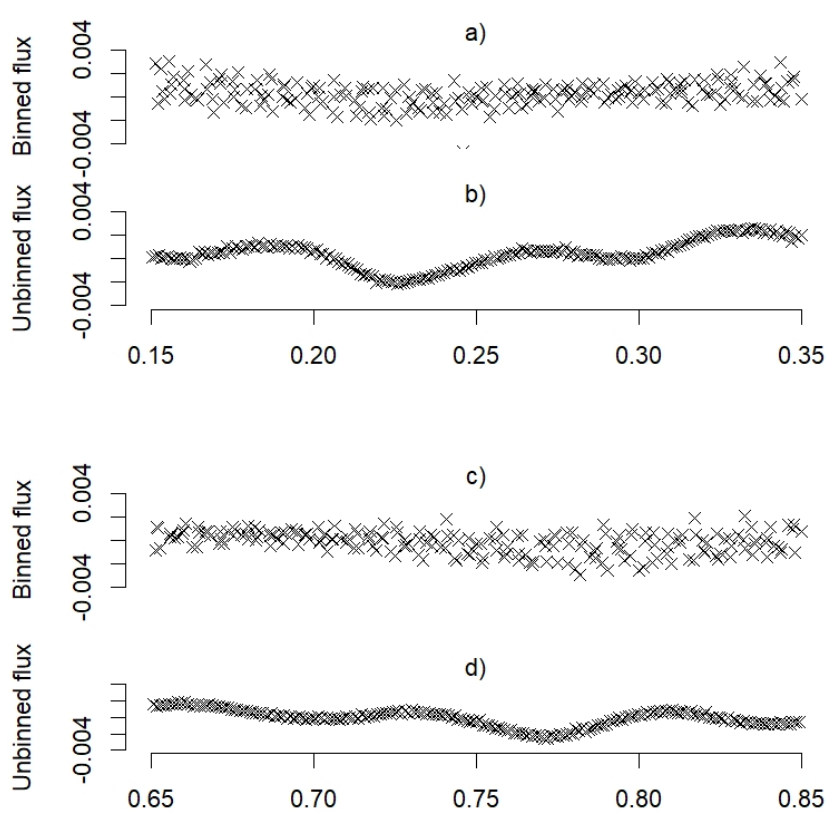

Figure 4. Panels (a) and (c) show the light-level of the out-ofeclipse phases resulting from the binning of the $14+$ individual TESS light curves of V Pup. This appears to have mostly smoothed out the intrinsic $\beta$ Cep variation visible in individual light curves, as shown in panels (b) and (d) (although traces of some predominating low-amplitude tendency can be seen in the binned data).

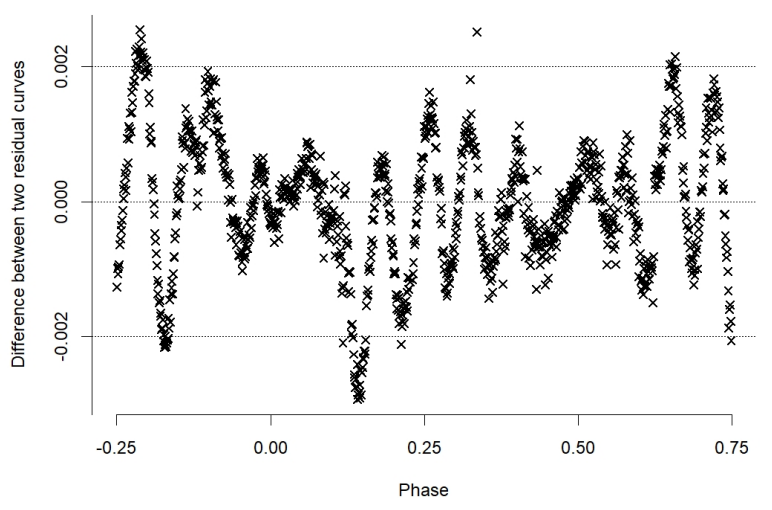

Figure 5. The character of the short-term oscillatory behaviour is confirmed by plotting the difference between the residuals from the single light curve fit and those of the 14 combined and binned light curves. The apparent damping out of the oscillator behaviour through the primary eclipse is indicative that it is the primary star which undergoes the pulsations, but this evidence, by itself, is not conclusive.
Table 2. Curve fitting results for TESS photometry of V Pup using WinFitTer 6.5. Parameters for which no error estimate is given are adopted from information separate to the fitting.

\begin{tabular}{lcc}
\hline & & \\
Parameter & Value & Error \\
\hline & & \\
$M_{2} / M_{1}$ & 0.50 & \\
$L_{1}$ & 0.518 & 0.002 \\
$L_{2}$ & 0.325 & 0.003 \\
$L_{3}$ & 0.162 & 0.004 \\
$r_{1}($ mean $)$ & 0.366 & 0.002 \\
$r_{2}($ mean $)$ & 0.307 & 0.002 \\
$i($ deg $)$ & 80.5 & 0.3 \\
$T_{h}(\mathrm{~K})$ & 26000 & \\
$T_{c}(\mathrm{~K})$ & 24000 & \\
$u_{11}$ & 0.25 & 0.01 \\
$u_{12}$ & 0.19 & 0.01 \\
$u_{21}$ & -0.03 & \\
$u_{22}$ & -0.03 & \\
$\Delta \phi_{0}($ deg $)$ & -2.04 & 0.02 \\
$\chi^{2} / \nu$ & 0.86 & \\
$\Delta l$ & 0.0015 & \\
\hline
\end{tabular}

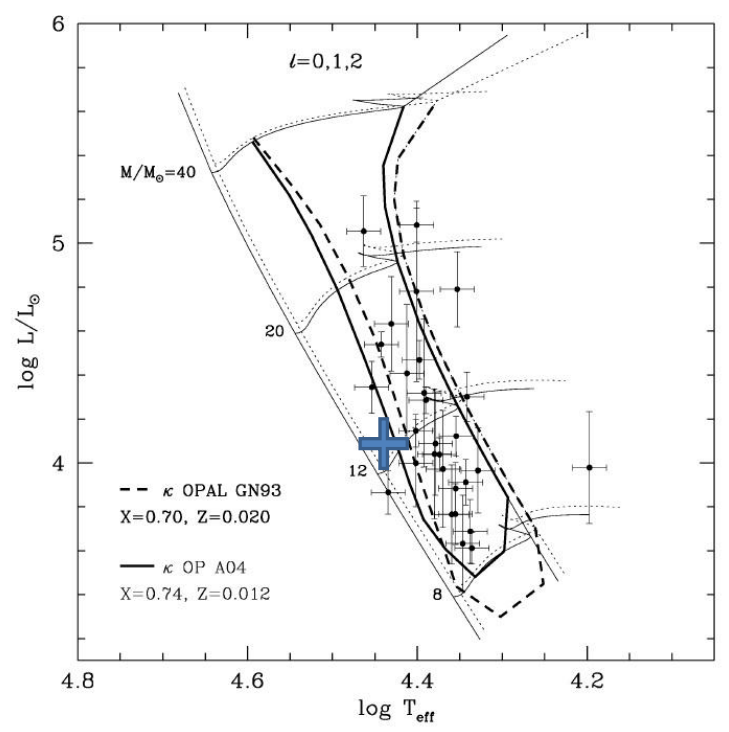

Figure 6. $\beta$ Cep stars in the HR diagram. The revised instability strip (copied from Fig 2 in Pamyatnykh, 2007), that uses improved OP opacities, is delineated by the thick-line border. The dense blue cross locates the primary of V Pup as an incipient $\beta$ Cep type variable in the $\mathrm{H}-\mathrm{R}$ diagram.

(Hearnshaw et al., 2002). This was attached to the $1 \mathrm{~m}$ McLellan telescope at the University of Canterbury Mt John Observatory (UCMJO) near Lake Tekapo ( 43 $\left.59^{\prime} \mathrm{S}, 174^{\circ} 27^{\prime} \mathrm{E}\right)$. Over 120 spectra of V Pup were collected during fairly clear weather during the period Feb 5-12, 2020.

The phase coverage of these UCMJO observations was 


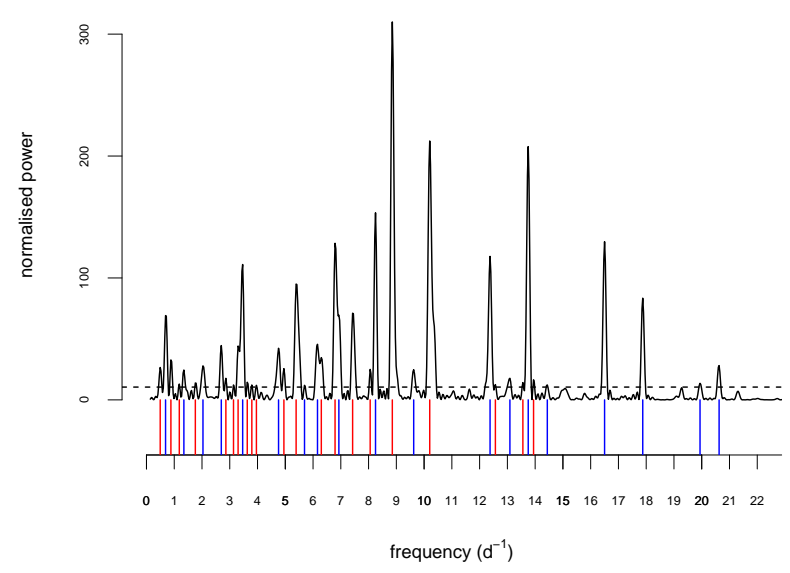

Figure 7. Lomb-Scargle periodogram of pulsational effects in the V Pup system. This may include a possible $\beta$-Cep type behaviour of the primary star. Solid blue lines indicate significant $(\mathrm{p}=0.01)$ frequencies that are within 0.1 of an integer multiple of the orbital frequency. Other significant frequencies are indicated by red lines. The two strongest signals are at $8.8555 \mathrm{~d}^{-1}(=2.71 \mathrm{~h})$ and 10.2030 $\mathrm{d}^{-1}(=2.35 \mathrm{~h})$.

about $65 \%$ of the complete cycle. Uncovered phases have been observed subsequently (TL) from the Saesteorra Observatory in the Wairarapa region of New Zealand $\left(41^{\circ} 13^{\prime} 07^{\prime \prime} \mathrm{S} ; 175^{\circ}\right.$ $27^{\prime} 51^{\prime \prime} \mathrm{E}$ ) with a 12 inch $\mathrm{f} / 8$ Ritchey-Chretien telescope. The spectroscopic equipment there consisted of a Lhires Littrow design spectrograph (Thizy, 2007), having a 35 micron slit and a 2400 lines/mm grating. This has a nominal resolution of approximately $\mathrm{R}=13400$. The spectral coverage was set to be from 6607 to $6813 \AA$, so as to encompass the He6678 line.

The UCMJO spectral images were collected with a $4 \mathrm{k} \times 4 \mathrm{k}$ Spectral Instruments (SITe) camera (Skuljan, 2004). The 100 $\mu$ fibre, which is suited to typical seeing conditions at $\mathrm{Mt}$ John, enables a theoretical resolution of $\sim 40000$. The regular image sequencing for wavelength and relative flux calibration was followed (cf. Blackford et al., 2019), however, an error in the detector's flux meter led to under-exposure of some calibration frames. Subsequent checks on telluric lines in the science frames provided assurance that our final data reductions were accurate and consistent. Exposure times were usually $\sim 100$ seconds for this 4 th mag star. The raw observations were reduced using an updated version of the software package HRSP (Skuljan, 2012), that produces wavelength calibrated and normalized output conveniently in FITS formatted files.

Some 45 clear orders of the échelle were set up for inspection. These have been studied using IRAF software. One of the IRAF subroutines (SPLOT), for example, allows image statistics to be checked. We could determine in this way a signal to noise ratio $(\mathrm{S} / \mathrm{N})$ for continuum pixel regions (away from flaws or telluric effects) to be usually of order 70 (with a $\sim 10 \%$ deterioration from orders 85 to 121 : the orders examined in this study). The spectra were also examined using the software package Visual Spec (VSPEC; Desnoux \& Buil, 2005), which is an MS-Windows ${ }^{\mathrm{TM}}$ based package that allows convenient analysis of spectra, including line fitting and identification functions.
Table 3. Identified spectral lines for V Pup ( $\mathrm{p} \equiv$ primary; s $\equiv$ secondary).

\begin{tabular}{ccll} 
Species & Order no. & Adopted $\lambda$ & Comment \\
He I & 85 & 6678.149 & well-defined p \& s \\
H $_{\alpha}$ & 87 & 6562.817 & strong, complex \\
He I & 97 & 5875.650 & well-defined p \& s \\
C II $\left(^{*}\right)$ & 110 & 5145.16 & p weak \\
He I & 112 & 5047.736 & weak, ill-defined p \& s \\
He I & 113 & 5015.675 & weak \\
He I & 115 & 4921.929 & strong p \& s \\
H & 117 & 4861.332 & strong, complex \\
He I & 121 & 4713.146 & weak p \& s \\
He II & 122 & 4685.7 & p only \\
C III+ OII & 122 & 4649.1 & strong blend \\
Si III & 125 & 4574.78 & edge of order \\
Si III & 125 & 4567.87 & blend \\
Si III & 125 & 4552.65 & blend \\
\hline
\end{tabular}

Table 4. Equivalent width measures of the $\lambda 6678$ He I feature for V Pup at the two elongations.

\begin{tabular}{ccccc} 
Phase & $\begin{array}{c}\text { Primary } \\
\text { ew }(\AA)\end{array}$ & $\begin{array}{c}\text { Error } \\
\AA\end{array}$ & $\begin{array}{c}\text { Secondary } \\
\text { ew }(\AA)\end{array}$ & $\begin{array}{c}\text { Error } \\
\AA\end{array}$ \\
0.25 & 0.64 & 0.04 & 0.52 & 0.08 \\
0.75 & 0.58 & 0.03 & 0.60 & 0.10 \\
\hline
\end{tabular}

The better-defined He I lines in Table 3 have a depth of $\sim 5 \%$ of the continuum and a width at base of up to $\sim 10$ $\AA$ or $\sim 350$ pixels. The lines hardly make a complete separation even at the highest observed elongation. The positioning of a well-formed symmetrical line (notably the primary's $\lambda 6678$ line) is consequently expected to be typically achieved to within $\sim 0.15 \AA$ or (equivalently) $\sim 7 \mathrm{~km} \mathrm{~s}^{-1}$. (The resulting distribution of $\sim 130$ measured RV displacements for either star is seen in Fig 8, while the He I $\lambda 6678$ feature itself is shown in Fig 9.)

VSPEC also allows the equivalent width $\left(\mathrm{W}_{\lambda}\right)$ of features to be directly read from its on-screen spectrometry bar. We could check, in this way, the spectral type assignations in the literature. Direct measures of equivalent widths have to be scaled against the relative luminosities of the two stars. The results of measuring a dozen pairs of the well-recorded $\lambda 6678$ He I feature at elongation are presented in Table 4.

These results are consistent with the B1V and B3IV literature types (cf. Leone \& Lanzafame, 1998; who provide effective temperatures that go with the type assignations), though the discrimination is not strong from these He I measures. The scatter of $\mathrm{W}_{\lambda}$ values indicates errors of $\sim 500 \mathrm{~K}$ in the assigned temperatures. There is some support for the approaching hemispheres to show slightly stronger absorptions (by $\sim 10 \%$ ) as discussed in previous papers (see Section 1.1).

\subsection{Radial velocities}

The RVs listed in Table 5 derived from profile fitting to determine the selected He I lines' centre-of-light wavelengths and 

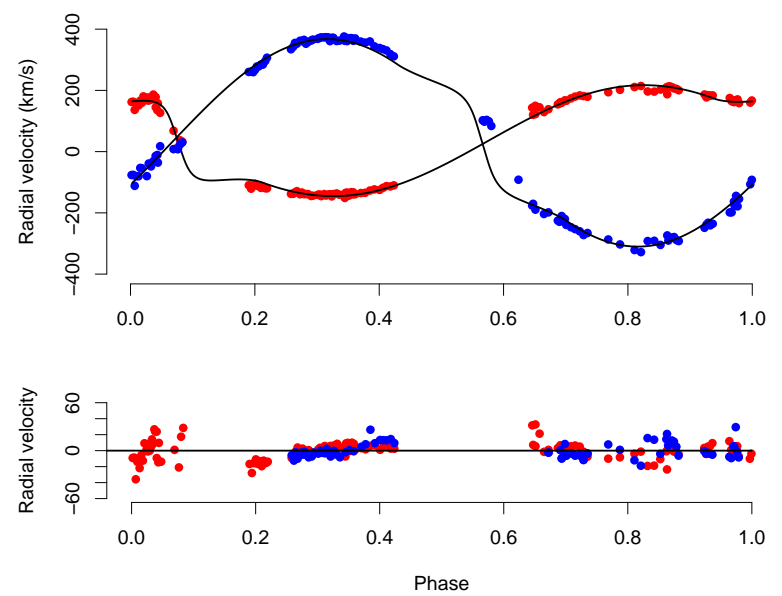

Figure 8. Orbital RV variation of the close binary V Pup (upper panel) with residuals (lower panel), (red for the primary, blue for the secondary). Full circles correspond to the UCMJO data; open circles to the data from the Saesteorra Observatory.

the corresponding Doppler displacements. The well-defined He I line at 6678 has been used mostly, with additional checks made with the He I 5876 line. The He I lines have the best $\mathrm{S} / \mathrm{N}$ ratios in our spectra, apart from the hydrogen lines that are difficult to model due to their highly blended wings. Each useful line was fitted separately at least three times and a final RV value obtained by averaging, noting the standard deviations in the same process.

The RVs in Table 5 have been corrected to solar system heliocentric values using HRSP and VSPEC data-reduction tools. The listed orbital phases are derived from the ephemeris given by Andersen et al. (1983), i.e.

$$
\text { MinI : HJD2445367.60633+1.4544859 E }
$$

This ephemeris is cited in the GCVS of Khopolov et al. (1987), and appears to be an accurate representation of early observations of the system. The adopted period is close to the mean value given by Kreiner et al. 2000, though it appears clear from more recent data that there is some variation in the period. This will be discussed in Section 5 .

Measured RVs are presented with an accuracy $0.1 \mathrm{~km} \mathrm{~s}^{-1}$, but that overestimates the accuracy of individual points (see above). Line-positioning depends mainly on the line properties, rather than the available spectrographic resolution, which is relatively high for HERCULES. But while RV measures are given with one significant decimal place in Table 5, equivalent to $\sim 0.1$ pixel at $\lambda 6678$, it can be seen in Fig 8 that the residuals are, in general, quite a bit greater than that. Some of these departures from the model appear to show systematic trending, but in the vicinity of the conjunctions blending introduces noticeable uncertainty into the measurement. For this reason the regions of the RV-curve fitted in order to define the velocity amplitudes in Table 6 were confined to phase regions outside the eclipse regions.

The binary's semi-major axis a can be determined from the RV amplitudes using the inclination obtained from the
Table 5. Radial velocity data for V Pup.

\begin{tabular}{|c|c|c|c|}
\hline $\begin{array}{c}\text { HJD } \\
2458880+\end{array}$ & $\begin{array}{c}\text { Orbital } \\
\text { phase }\end{array}$ & $\begin{array}{c}\mathrm{RV} 1 \\
\mathrm{~km} \mathrm{~s}^{-1}\end{array}$ & $\begin{array}{c}\mathrm{RV} 2 \\
\mathrm{~km} \mathrm{~s}^{-1}\end{array}$ \\
\hline 5.0856 & 0.6475 & 120.4 & -170.4 \\
\hline 5.0909 & 0.6512 & 122.5 & -189.5 \\
\hline 5.1434 & 0.6873 & 154.4 & -225.2 \\
\hline 5.1473 & 0.6899 & 157.2 & -229.2 \\
\hline 5.8750 & 0.1902 & -109.6 & 260.3 \\
\hline 5.8804 & 0.1940 & -121.1 & 260.9 \\
\hline 5.8852 & 0.1973 & -109.2 & 259.5 \\
\hline 5.8897 & 0.2004 & -105.1 & 267.4 \\
\hline 5.8938 & 0.2032 & -111.6 & 278.0 \\
\hline 5.8984 & 0.2063 & -109.6 & 278.4 \\
\hline 5.9034 & 0.2098 & -118.4 & 284.5 \\
\hline 5.9079 & 0.2129 & -113.8 & 284.3 \\
\hline 5.9134 & 0.2167 & -119.5 & 295.8 \\
\hline 5.9176 & 0.2195 & -119.9 & 306.8 \\
\hline 6.0978 & 0.3434 & -140.6 & 376.0 \\
\hline 6.1013 & 0.3458 & -134.8 & 372.7 \\
\hline 6.1050 & 0.3484 & -133.8 & 361.0 \\
\hline 6.1095 & 0.3515 & -143.6 & 370.4 \\
\hline 6.1149 & 0.3552 & -132.3 & 370.8 \\
\hline 6.1195 & 0.3584 & -133.0 & 362.0 \\
\hline 6.1240 & 0.3614 & -139.1 & 369.6 \\
\hline 6.8565 & 0.8651 & 212.5 & -290.5 \\
\hline 6.8601 & 0.8675 & 213.1 & -283.9 \\
\hline 6.8655 & 0.8712 & 210.2 & -280.8 \\
\hline 6.8700 & 0.8743 & 206.4 & -279.0 \\
\hline 6.8757 & 0.8783 & 204.8 & -289.2 \\
\hline 6.8808 & 0.8818 & 200.4 & -292.1 \\
\hline 6.9411 & 0.9232 & 186.6 & -248.8 \\
\hline 6.9456 & 0.9263 & 177.1 & -238.4 \\
\hline 6.9495 & 0.9290 & 184.4 & -232.3 \\
\hline 6.9546 & 0.9325 & 182.6 & -240.7 \\
\hline 6.9602 & 0.9364 & 183.7 & -235.6 \\
\hline 7.0098 & 0.9705 & 161.7 & -163.2 \\
\hline 7.0150 & 0.9740 & 164.8 & -144.5 \\
\hline 7.0184 & 0.9764 & 170.6 & -178.6 \\
\hline 7.0219 & 0.9788 & 158.4 & -154.4 \\
\hline 7.0481 & 0.9968 & 160.1 & -106.6 \\
\hline 7.0518 & 0.9993 & 166.9 & -92.1 \\
\hline 7.0553 & 0.0017 & 162.1 & -76.4 \\
\hline 7.0591 & 0.0044 & 163.1 & -76.1 \\
\hline 7.0626 & 0.0068 & 136.3 & -111.8 \\
\hline 7.0662 & 0.0092 & 158.4 & -80.2 \\
\hline 7.0716 & 0.0129 & 150.6 & -80.5 \\
\hline 7.0756 & 0.0157 & 166.6 & -53.2 \\
\hline 7.0792 & 0.0182 & 159.3 & -54.2 \\
\hline 7.1155 & 0.0431 & 135.3 & -11.0 \\
\hline 7.8768 & 0.5665 & - & 102.3 \\
\hline 7.8805 & 0.5691 & - & 97.8 \\
\hline 7.8859 & 0.5728 & - & 103.6 \\
\hline 7.8912 & 0.5764 & - & 99.1 \\
\hline 7.8968 & 0.5803 & - & 83.8 \\
\hline 7.9603 & 0.6240 & - & -91.8 \\
\hline 7.9924 & 0.6460 & 143.4 & -176.2 \\
\hline 7.9998 & 0.6511 & 149.7 & - \\
\hline 8.0095 & 0.6578 & 144.8 & - \\
\hline 8.0002 & 0.6650 & 129.1 & -203.7 \\
\hline 8.0304 & 0.6721 & 138.4 & -198.4 \\
\hline 8.0614 & 0.6935 & 159.5 & -210.3 \\
\hline 8.0721 & 0.7008 & 165.9 & -239.4 \\
\hline 8.0831 & 0.7084 & 173.9 & -246.9 \\
\hline 8.0928 & 0.7150 & 179.8 & -253.1 \\
\hline
\end{tabular}


Table 5. Radial velocity data for V Pup (continued).

\begin{tabular}{|c|c|c|c|}
\hline $\begin{array}{c}\text { HJD } \\
2458880+\end{array}$ & $\begin{array}{c}\text { Orbital } \\
\text { phase }\end{array}$ & $\begin{array}{c}\mathrm{RV} 1 \\
\mathrm{~km} \mathrm{~s}^{-1}\end{array}$ & $\begin{array}{c}\mathrm{RV} 2 \\
\mathrm{~km} \mathrm{~s}^{-1}\end{array}$ \\
\hline 8.1036 & 0.7225 & 183.7 & -260.2 \\
\hline 8.1125 & 0.7286 & 181.4 & -272.4 \\
\hline 8.1218 & 0.7350 & 179.2 & -265.8 \\
\hline 8.8826 & 0.2581 & -138.0 & 334.3 \\
\hline 8.8879 & 0.2617 & -137.8 & 342.6 \\
\hline 8.8919 & 0.2645 & -132.9 & 355.0 \\
\hline 8.8965 & 0.2676 & -129.2 & 352.5 \\
\hline 8.9039 & 0.2727 & -139.3 & 362.1 \\
\hline 8.9082 & 0.2757 & -133.6 & 360.9 \\
\hline 8.9130 & 0.2790 & -138.8 & 365.1 \\
\hline 8.9177 & 0.2822 & -138.4 & 352.4 \\
\hline 8.9226 & 0.2856 & -138.7 & 358.2 \\
\hline 8.9298 & 0.2905 & -144.8 & 362.8 \\
\hline 8.9338 & 0.2933 & -144.0 & 365.9 \\
\hline 8.9450 & 0.3010 & -141.3 & 368.4 \\
\hline 8.9491 & 0.3038 & -139.8 & 371.2 \\
\hline 8.9533 & 0.3067 & -145.3 & 373.2 \\
\hline 8.9603 & 0.3115 & -138.6 & 373.1 \\
\hline 8.9646 & 0.3144 & -143.4 & 373.7 \\
\hline 8.9692 & 0.3176 & -140.5 & 373.4 \\
\hline 8.9739 & 0.3208 & -139.3 & 360.9 \\
\hline 8.9781 & 0.3237 & -146.2 & 371.8 \\
\hline 8.9819 & 0.3263 & -138.8 & 371.8 \\
\hline 8.9894 & 0.3315 & -136.6 & 361.2 \\
\hline 8.9958 & 0.3359 & -140.7 & 362.5 \\
\hline 9.0086 & 0.3447 & -151.3 & 362.0 \\
\hline 9.0173 & 0.3507 & -141.2 & 367.9 \\
\hline 9.0281 & 0.3581 & -139.6 & 367.3 \\
\hline 9.0461 & 0.3705 & -135.3 & 359.4 \\
\hline 9.0560 & 0.3773 & -134.4 & 356.1 \\
\hline 9.0673 & 0.3850 & -127.1 & 359.9 \\
\hline 9.0778 & 0.3923 & -123.4 & 343.8 \\
\hline 9.0899 & 0.4006 & -124.9 & 338.5 \\
\hline 9.0978 & 0.4060 & -118.3 & 334.7 \\
\hline 9.1060 & 0.4117 & -112.7 & 330.4 \\
\hline 9.1151 & 0.4179 & -113.6 & 318.5 \\
\hline 9.1230 & 0.4235 & -110.2 & 311.2 \\
\hline 9.9090 & 0.9638 & 175.2 & -198.6 \\
\hline 9.9135 & 0.9668 & 164.7 & -198.3 \\
\hline 9.9205 & 0.9716 & 167.6 & -177.1 \\
\hline 10.0000 & 0.0263 & 167.9 & -80.1 \\
\hline 10.0043 & 0.0293 & 166.9 & -38.9 \\
\hline 10.0096 & 0.0329 & 178.0 & -48.3 \\
\hline 10.0151 & 0.0367 & 186.4 & -31.3 \\
\hline 10.0205 & 0.0404 & 178.3 & -14.2 \\
\hline 10.0257 & 0.0440 & 158.0 & -35.8 \\
\hline 10.0311 & 0.0477 & 126.7 & 17.7 \\
\hline 10.0624 & 0.0692 & 68.4 & 8.2 \\
\hline 10.0727 & 0.0763 & 14.5 & 7.8 \\
\hline 10.0778 & 0.0798 & 37.0 & 23.5 \\
\hline 10.0829 & 0.0833 & 32.4 & 30.1 \\
\hline 10.9672 & 0.6913 & 161.3 & -226.4 \\
\hline 10.9779 & 0.6986 & 167.6 & -219.9 \\
\hline 83.803 & 0.7684 & 193.9 & -298.4 \\
\hline 83.831 & 0.7873 & 201.9 & -314.6 \\
\hline 83.865 & 0.8107 & 211.0 & -333.0 \\
\hline 83.880 & 0.8210 & 214.4 & -339.7 \\
\hline 83.896 & 0.8318 & 196.5 & -303.6 \\
\hline 83.911 & 0.8424 & 196.0 & -302.7 \\
\hline 83.926 & 0.8524 & 202.7 & -316.2 \\
\hline 83.941 & 0.8633 & 187.4 & -285.2 \\
\hline
\end{tabular}

Table 6. Out-of-eclipse curve fitting results for measured MJUO RVs for the close binary system V Pup. Symbols have their usual meanings.

\begin{tabular}{|c|c|c|}
\hline Parameter & Value & Error \\
\hline$K_{1} \sin i\left(\mathrm{~km} \mathrm{~s}^{-1}\right)$ & 175.4 & 3.2 \\
\hline$K_{2} \sin i\left(\mathrm{~km} \mathrm{~s}^{-1}\right)$ & 338.8 & 5.4 \\
\hline$\Delta \phi_{0}(\operatorname{deg})$ & 23.6 & 1.2 \\
\hline$V_{\gamma}\left(\mathrm{km} \mathrm{s}^{-1}\right)$ & 28.9 & 1.5 \\
\hline$\nu$ & $74(\mathrm{p}) \quad 63(\mathrm{~s})$ & \\
\hline$\chi^{2} / \nu$ & $1.18(\mathrm{p}) \quad 1.05$ & \\
\hline$\sigma\left(\mathrm{km} \mathrm{s}^{-1}\right)$ & $7(\mathrm{p}) \quad 9(\mathrm{~s})$ & \\
\hline
\end{tabular}

Table 7. Line-modelling parameters for V Pup at elongation.

\begin{tabular}{lccc}
\hline \multicolumn{1}{c}{ Parameter } & 1st elong. & 2nd elong. & Error \\
\hline & & & \\
$I_{0,1}$ & -0.059 & -0.047 & 0.002 \\
$I_{0,2}$ & -0.040 & -0.036 & 0.002 \\
$\lambda_{1}$ & 6676.78 & 6684.09 & 0.06 \\
$\lambda_{2}$ & 6687.89 & 6673.69 & 0.09 \\
$v_{\text {rot }, 1 \mathrm{~km} \mathrm{~s}^{-1}}$ & 218 & 246 & 5 \\
$v_{\mathrm{rot}, 2 \mathrm{~km} \mathrm{~s}^{-1}}$ & 187 & 209 & 6 \\
$s_{1} \mathrm{~km} \mathrm{~s}^{-1}$ & 8 & 7 & 2 \\
$s_{2} \mathrm{~km} \mathrm{~s}^{-1}$ & 17 & 12 & 5 \\
$\Delta f$ & 0.015 & 0.015 & \\
$\chi^{2} / \nu$ & 1.2 & 1.3 & \\
\hline
\end{tabular}

photometric analysis. We have,

$$
a=\frac{P\left(K_{1}+K_{2}\right) \sqrt{1-e^{2}}}{2 \pi \sin i},
$$

where symbols have their usual meanings (Smart, 1960). On substitution of the foregoing values for the Eqn 1 period $P$ (in seconds) and the Table $6 \mathrm{RV}$ amplitudes $K_{1}$ and $K_{2}$, and adopting the eccentricity $e$ to be negligible, we find $a \approx$ $1.043 \times 10^{7} \mathrm{~km}$, or $14.96 \mathrm{R}_{\odot}$. Using the relative radii from Table 2 the mean radii turn out to be 5.48 and $4.59 \mathrm{R}_{\odot}$.

\subsection{Rotational velocities}

The profiles of the He I lines, particularly the 6678 feature, are well-defined and capable of yielding rotational and turbulence parameters. We show the results of such profile fitting in Table 7 and Fig 9.

The entries in Table 7 follow a similar arrangement to the other tables of model-fitting in this paper. The quantities $I_{0}$ correspond to the central depths on the same relative scale. Mean wavelengths $\lambda_{1,2}$ are self-explanatory. The rotational velocities $v_{\text {rot, } 1,2}$ are calculated with an inclination $i=80.5^{\circ}$ (Table 2) to apply a correction for the projection. The parameters $s_{1,2}$ represent the widths of the gaussian broadening that is convolved with the rotational broadening. These may be interpreted as a measure of the turbulence in the source. The secondary appears relatively more disturbed in this way than the primary, perhaps reflecting its Roche lobe instability condition.

The angular velocity of the system, using the period given 

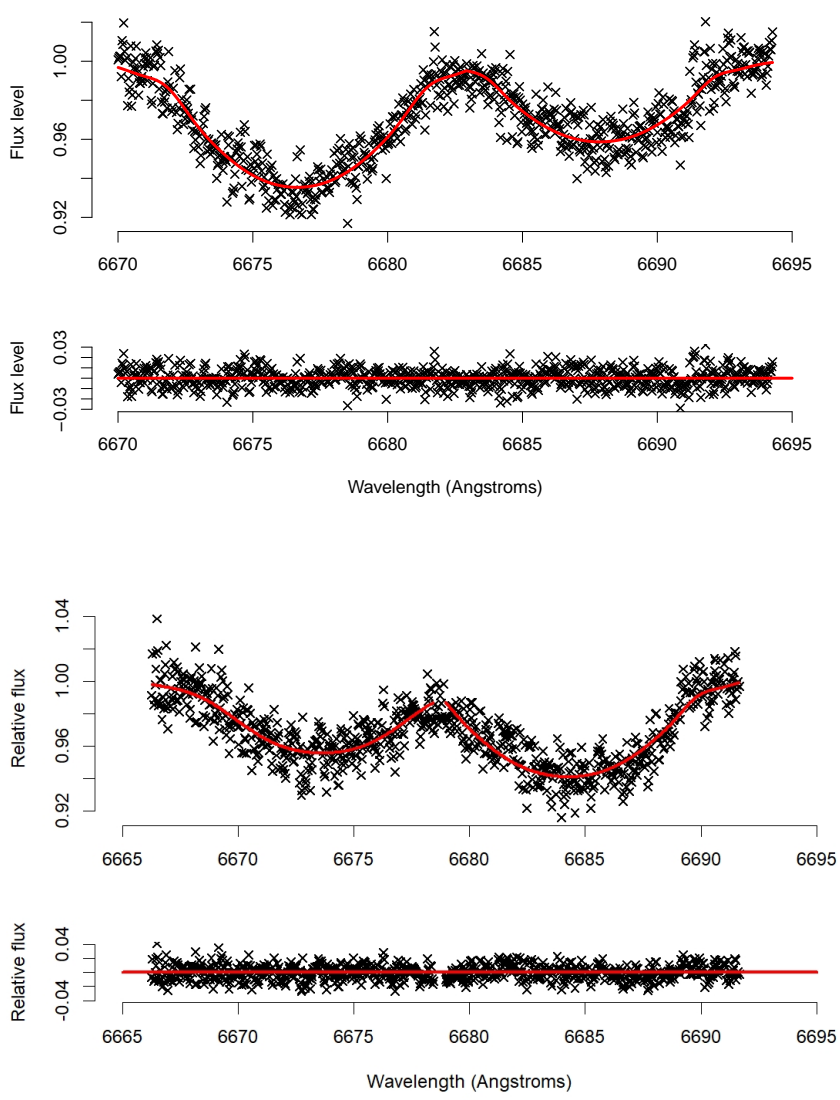

Figure 9. Profile fitting to the HeI $\lambda 6678$ lines: (upper pair) 1st elongation (primary approaching), with residuals shown beneath; (lower pair) 2nd elongation(primary receding), residuals below. The lines have been fitted separately and do not quite return to the continuum level between the absorption features: the fitting thus shows a discontinuity in that region.

in Section 2, is $5.00 \times 10^{-5}$ radians $\mathrm{s}^{-1}$. With the stellar radii given in the previous section, mean synchronized rotation speeds $(\omega R)$ then turn out to be 191 and $160 \mathrm{~km} \mathrm{~s}^{-1}$. These speeds are appreciably less than the measured values in Table 7 , though they are in the same ratio $(\sim 0.84)$, implying an excess of the equatorial speed above synchronism of about $15 \%$. This is comparable to the distortions from sphericity of these rapidly rotating spheroids (Fig 3). Theoretical timescales for synchronization ( 1 Myr; Zahn, 1977) are short enough to expect a synchronized configuration.

Rossiter effects can be seen in the data, but the measures for the secondary are too poor (mainly as a result of blending) to be included in effective fitting trials. It was intended to check the rotational velocity of the primary by fitting the radial velocity curve in the eclipse region, since the distortion from the more nearly sinusoidal variation through the uneclipsed phases shows a clearly visible effect in Fig 8. This proved ineffective, however, as the difference between the results of setting $v_{\text {rot }, 1}$ (sync) and $v_{\text {rot }, 1}(2 \times$ sync $)$ on the Rossiter effect is barely noticeable against the scatter in the relevant phase range ( 0.0 to 0.1 in Fig 8$)$.
Table 8. Absolute parameters of V Pup system from combined results of data fittings. The column of comparison numbers come from the work of Andersen et al. (1983). There is closer agreement between the parameters of the present paper and those of the comparison than with the other papers cited by Andersen et al., though the formal errors given by the latter for the radii and masses are more stringent than ours (see text). On the other hand, our estimates of the $V$ magnitudes, given with lower uncertainties, produce a significantly closer photometric parallax to the independent HIPPARCOS value.

\begin{tabular}{lcccc}
\hline & & & & \\
Parameter & Value & Error & Comparison & Error \\
\hline & & & & \\
$P \mathrm{~d}$ & 1.4544859 & - & - & - \\
$a(\mathrm{R} \odot)$ & 14.96 & 0.2 & 15.27 & 0.09 \\
$R_{1} \odot$ & 5.48 & 0.18 & 6.18 & 0.07 \\
$R_{2} \odot$ & 4.59 & 0.15 & 4.90 & 0.05 \\
$T_{1} \mathrm{~K}$ & 26000 & 1000 & 28200 & 1000 \\
$T_{2} \mathrm{~K}$ & 24000 & 1000 & 26600 & 1000 \\
$M_{1} \odot$ & 14.0 & 0.5 & 14.86 & 0.24 \\
$M_{2} \odot$ & 7.3 & 0.3 & 7.76 & 0.14 \\
$\log L_{1} \odot$ & 4.10 & 0.08 & 4.34 & 0.06 \\
$\log L_{2} \odot$ & 3.81 & 0.08 & 4.04 & 0.07 \\
$V_{1} \mathrm{mag}$ & 5.10 & 0.05 & 4.83 & 0.16 \\
$V_{2} \mathrm{mag}$ & 5.59 & 0.07 & 5.50 & 0.16 \\
$V_{\gamma}(\mathrm{km} / \mathrm{s})$ & 20.0 & 1.1 & 13.2 & 2.5 \\
$\operatorname{distance~pc}$ & 320 & 10 & 380 & $-30-$ \\
age Myr & 5 & 0.8 & & \\
\hline
\end{tabular}

\section{ABSOLUTE PARAMETERS}

The foregoing orbital semi-major axis and radii of components are listed in Table 8. Using the HIPPARCOS period $(0.0039822 \mathrm{yr})$ with the value of $a$ as $0.06961 \mathrm{AU}$, we find the sum of the close binary components' masses directly from Kepler's third law to be $21.27 \mathrm{M}_{\odot}$. Given the mass ratio $q=K_{1} / K_{2}=0.518$, we then find the listed individual masses, which are sensitive to the derived RV amplitude and inclination parameters. The absolute radii, $R_{1,2}$ that come from multiplying the relative radii of Table 2 by $a$, are determined with higher precision than the masses. These radii, together with the temperatures from Table 2, produce the luminosities given in Table 8.

The set of comparison parameters in Table 8 come from the work of Andersen et al. (1983). There is a good general agreement between our derived parameters and and those of Andersen et al., though the formal errors given by the latter for the radii and masses are about twice as low as ours. The differences on the mass values come mainly from the analysis of the radial velocity curves. In Section 3 we gave reasons why individual RV measures should have errors on the order of $10 \mathrm{~km} \mathrm{~s}^{-1}$, and this would also appear to apply to the measures listed by Andersen et al. (their Table 1), where the average datum error is close to $10 \mathrm{~km} \mathrm{~s}^{-1}$. Andersen et al. (1983) corrected their initial RV measures according to the prescription of a selected model. It is seen in Table 3 of Andersen et al. (1983) that the differences between their initial and corrected model results are significantly greater than the formal errors of the modelling parameters, while our radial velocity amplitudes (Table 6) are between the two parameter sets produced by Andersen et al. An empirical stance with 


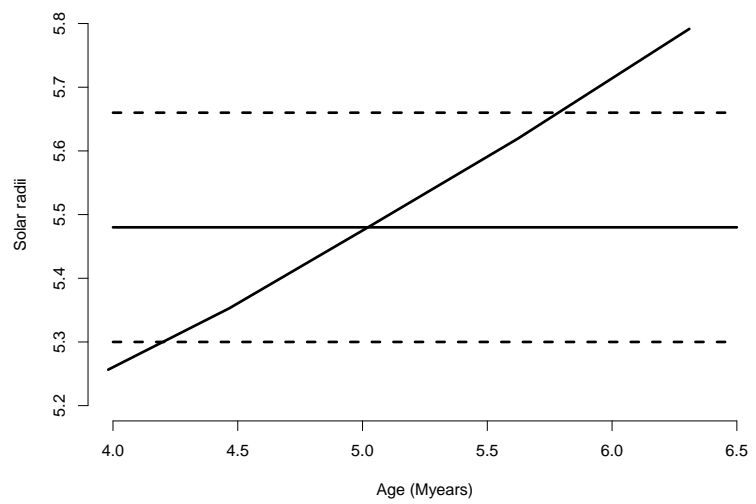

Figure 10. Increasing radius of a $14 \mathrm{M}_{\odot}$ star is shown as a function of age from the Padova models (Marigo et al., 2017), together with the determined radius (horizontal line) and its uncertainty limits (dotted). An age of about $5 \mathrm{Myr}$ is deduced.

regard to deviations of data from modelling predictions then allows that the real errors of parameters may be appreciably greater than the formal ones of a particular modelling result. Our more conservative assessment of the modelling accuracy comes after various curve-fitting experiments with different assumptions on the non-adjustable parameters.

The photometric parallax $\pi$ is derived from the formula (Budding \& Demircan, 2007; Eqn 3.42)

$$
\log \pi=7.454-\log R-0.2 V-2 F^{\prime}{ }_{V},
$$

where $R$ is the absolute radius, $V$ the visual magnitude and $F^{\prime}{ }_{V}$ the flux parameter $\left(=\log T_{e}-0.1 \mathrm{BC} ; T_{e}\right.$ being the effective temperature and $\mathrm{BC}$ the corresponding bolometric correction). The reciprocal of $\pi$ would give the distance $\rho$ to be $360 \pm 12$ pc, but Eqn 3 refers to a $V$ magnitude without interstellar extinction. The adopted $B-V$ colour of -0.17 for the components (Ducati, 2002), when compared with the representative unreddened colour of -0.27 for this pair of early B-type stars (Budding \& Demircan, 2007, Table 3.6) yields a colour excess of 0.10 . From this, we can estimate an interstellar absorption value of $A_{V}=0.30$ (Cardelli et al., 1989). The effect of the absorption implies the distance would be reduced by $\sim 11 \%$, leading to the value listed in Table 8 , which is in keeping with the derived scale of interstellar extinction for a near-Disk source. This distance is within the error limit of the value given by HIPPARCOS (295 $\pm 30 \mathrm{pc})$.

Table 8 also gives and age estimate, that is determined from matching the calculated radius of the MS component with Padova evolutionary models (Fig 10). This result is discussed further in Section 6.

\section{O - C EFFECTS}

If we plot the difference between observed times of minima for V Pup and those predicted by the reference ephemeris of Kreiner et al. (2000) (Fig 11), i.e.

$$
\text { Min : I = HJD2428648.2837+ 1.45448686E , }
$$

we can see directly the general parabolic trend pointed out by Qian et al. (2008). It is worth noting the O-C Gateway

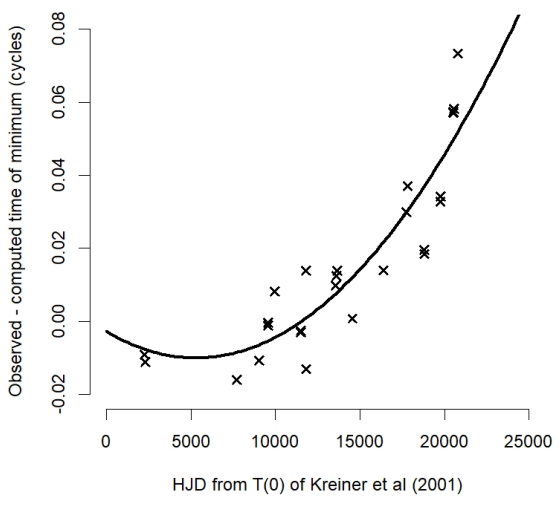

Figure 11. Parabolic trend in the $\mathrm{O}-\mathrm{C}$ eclipse timings of V Pup using the ephemeris of Kreiner et al. (2000).

revision however ${ }^{1}$, where a longer mean period also gives a reasonable fit if we admit somewhat larger timing uncertainties. Our procedure is a little different to that of Qian et al., in that we have added some more recent times of minima and separated out the parabolic trend before addressing the residuals, but our result is fairly similar. We find a second order term in the epoch number $E$ of order $3.5 \times 10^{-10}$, a slight reduction in the mean period from that given by Kreiner et al. (2000) of around $5 \times 10^{-6}$ and a slight positive shift in the reference epoch of about 10 minutes. The uncertainties in these parameters are on the order of $10 \%$ of their values. The implied rate of period variation is $\dot{P} \approx 1.8 \times 10^{-7} \mathrm{~d} \mathrm{yr}^{-1}$; slightly less than that found by Qian et al., but within the error measures of the determinations.

From a close examination of the residuals from the parabolic fitting, Qian et al. (2008) found an additional sinusoidal variation as a $2.5 \sigma$ effect, with a period of $\sim 1997 \mathrm{~d}$. We can see this effect clearly in Fig 12 for the first 17 points discussed by Qian et al. However, after adding in the more recent data from Kreiner ${ }^{2}$; Skarka (Hon̆ková et al. 2013); the BRITE collaboration (Popowicz et al., 2017) and TESS (Ricker et al., $2015)$; the fit significantly deteriorates. These times of minima have been listed by MB on the VSS website ${ }^{3}$. According to the ephemeris of Kreiner et al. (2000), given as Eqn 4, the mean time of inferior conjunction for the radial velocity curve shown in Fig 8 should have occurred at HJD 2458888.520 the 20791st such conjunction after the reference epoch. As can be seen in Fig 8, the conjunction was a little later than this, by $0.107 \pm 0.007 \mathrm{~d}(0.073$ cycles $)$. This has been added to Fig 11 as the rightmost point.

Let us assume that the times of minimum number $E$, that is $T(E)$, follow the parabolic form

$$
T(E)=A+B E+C E^{2}
$$

The period at $E$, usually given in days, is then given by the

\footnotetext{
1 http://var2.astro.cz/ocgate/ocgate.php?star $=\mathrm{V}+\mathrm{Pup}$ \&submit $=$ Submit\&lang $=$ en

2 https://www.aavso.org/bob-nelsons-o-c-files

3 https://www.variablestarssouth.org/budding-et-al-v-puppis/
} 


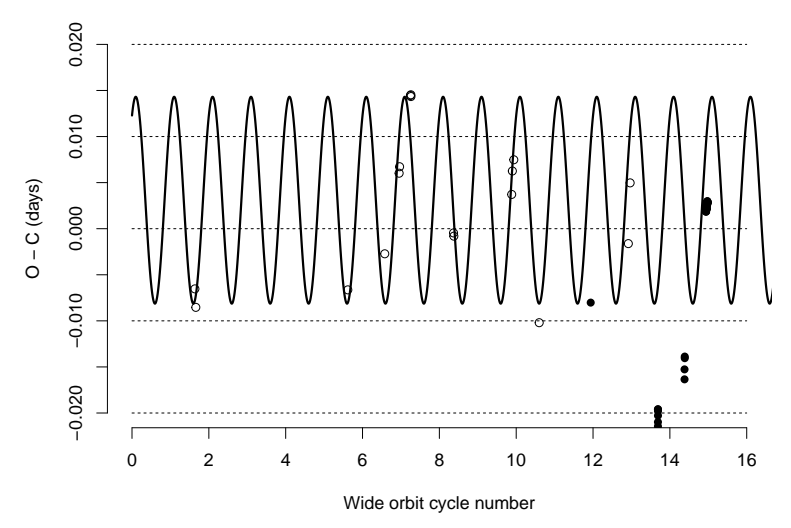

Figure 12. Sinusoidal effect in the $\mathrm{O}-\mathrm{C}$ eclipse residuals after removing the parabolic trend in Fig 11.17 selected points (open circles) conform well to the wide orbit period (5.47 yr) of Qian et al. (2008). Later values (filled circles), however, include some distinct discrepancies with the sinusoidal model (see text).

uniformly increasing slope

$$
P(E)=B+2 C E,
$$

where, in practice, $C$ is very small compared to $B$.

After selecting some reference epoch $T_{0}$ where $E=E_{0}=0$, the $\mathrm{O}-\mathrm{C}$ diagram is formed from the difference between $T(E)$ and corresponding $T$-values calculated along the line $T_{c}=P_{c} E+$ const., where $P_{c}=B_{c}$, say, and the constant term in the expression for $T_{c}$ is $A_{c}$, say. The constants $A_{c}$, $B_{c}$ would be assigned to give the best position of the line to match the observed trend in the given $E, T$ interval. We would expect them to be close to $A$ and $B$ in practice. The $\mathrm{O}-\mathrm{C}$ values are then

$$
\mathrm{O}-\mathrm{C}=T(E)-T_{c}=\left(A-A_{c}\right)+\left(B-B_{c}\right) E+C E^{2},
$$

in days. This is again of parabolic form, and with the same coefficient of the $E^{2}$ term. Reasonably measurable values of $C$, for an $\mathrm{O}-\mathrm{C}$ of order $0.1 \mathrm{~d}$ or greater, involve a suitably covered interval with a half-range for $E$ reaching to $\sim 10000$ periods, i.e. $C$ should be typically greater than about $10^{-9}$ for a very confident determination.

The change of period $\Delta P$ with each event $(\Delta E=1)$ becomes $d P / d E=2 C$. and the rate of change of period per year at $E$ is then $730.5 C / P(E)$. The amount of mass lost per year $\Delta M_{1}$, follows as (Kreiner \& Ziółkowski, 1978),

$$
\Delta M_{1}=243.3 \frac{M_{1} C}{g(x) P^{2}} \mathrm{yr}^{-1},
$$

where $g(x)$ is a function of the mass ratio $x=M_{1} /\left(M_{1}+M_{2}\right)$ that depends on what happens to the matter, as a whole, shed by the mass-losing star, here identified as $M_{1}$ - the originally more massive component. In the case where total mass and angular momentum of the binary are conserved $g(x)$ can be shown to equal $(2 x-1) /(1-x)$; so its value would be currently close to -0.5 , from the data in Table 8 . We then derive a mass transfer rate of about $6 \times 10^{-7}$ solar masses per year. If most of the mass is lost from the system, then at low $M_{1}$, $g(x) \rightarrow-1$; so a lower mass loss rate, say $\sim 3 \times 10^{-7}$ solar masses per year, would achieve the same period increase (i.e. a lower $C$ in Eqn 8).

In fact, we can deduce that, if the age of the system is only $\sim 5 \mathrm{Myr}$ and the erstwhile primary must have been bigger than half the current mass of the system, the average rate of mass loss of that star must be at least $\sim 10^{-6} \mathrm{M}_{\odot} \mathrm{yr}^{-1}$, and probably more than that, in keeping with the lack of appreciable separation of the stars that would have occurred with the decline to a mass ratio of 0.5 in a conservative regime.

We can compare the mass loss rate with what follows from the mass loss formula (cf. Awadalla \& Budding, 1982; Murad \& Budding, 1984):

$$
\dot{M}_{1} \approx-3 \eta s M_{1} / R_{1}
$$

where we can take the mass-losing star's mass and radius $\left(M_{1}\right.$ and $R_{1}$ ) from (Table 8 ) and give consideration to the relative density parameter $\eta$ and rate of surface expansion $s$. The value $s$ might be considered on the basis of a Case A mass-transfer model, in which the loser remains in hydrostatic equilibrium as a Main Sequence star but with a modified evolution timescale. That would allow it to be compared with a 7.3 solar mass star of age close to $28 \mathrm{Myr}$. The corresponding value of $s$ then works out as $1.0 \times 10^{-7}$ solar radii per year, using the Padova evolution models (Marigo et al., 2017). $\operatorname{Eqn}(9)$ then yields

$$
\dot{M}_{1} \approx-5 \eta \times 10^{-7}
$$

solar masses per yr. The parameter $\eta=\rho_{s} / \bar{\rho}$ : the ratio of the density in the subphotospheric layer where the predominating systematic motion is the outward expansion, to the mean density of the star as a whole, appears to be relatively small. Using continuity of the flow, we can write $\rho_{s}=\rho_{R 1} v_{R 1} \epsilon^{2} / s$, where $v_{R 1}$ is the velocity of sound in the atmospheric layers where the mean density is $\rho_{R 1}$, and $\epsilon=\Omega a / v_{R 1}=h / 2 R_{1} \approx$ $1 / 100, \Omega$ being the system's angular velocity and $h$ the masstransferring stream's width (cf. Lubow \& Shu, 1975). With $v_{R 1}$ estimated at $6 \times 10^{-9}(\mathrm{Cox}, 2000)$, we find $\eta \approx 0.03$.

In other words, if this Case A mass loser were to behave like a Main Sequence star of the same mass and luminosity, the calculated mass loss rate is more than an order of magnitude too low to account for the apparent rate of period increase. The star is required to expand much more rapidly than that.

Of course, this follows if we take into account an originally high mass of the mass-losing star. That would entail the earlier development of a more massive core than would correspond with a star that remained at $7 \mathrm{M}_{\odot}$. Also, $\eta$ is larger at earlier stages of interactive evolution, as can be seen from the $\epsilon$ parameter, in the Lubow and Shu (1975) discussion, being greater as a result of both increased mass and proximity. If we adopt $\eta=0.1$ and use the Padova evolution tables with an original value of $M_{1}$ of around $15 \mathrm{M}_{\odot}$, we find an expansion approaching $1.0 \times 10^{-6}$ solar radii per year within the time-frame of a few million years. Eqn (9) then allows a fair agreement between the amount of mass lost by $M_{1}$ and a Case A model for the binary evolution.

\section{CONCLUSIONS}

The V Pup system has been long recognized as a benchmark example for studies of massive close binary Algol-type evolution. It may be compared with $\mu^{1}$ Sco, where Budding et al. 
(2015) found that the primary was fairly consistent with the properties of a single star of its age and mass. In turn, that implies that the matter shed by the over-luminous secondary was probably mostly ejected from the system rather than transferred, thus taking angular momentum out of the orbit and keeping the pair in relative close proximity. This also appears to be the case for $\mathrm{V}$ Pup. This is then the regular Case A situation discussed by Andersen et al. (1983), whose Fig 6 shows the primary close to the trend of the Main Sequence for young massive stars as quantified by Popper (1980), while the secondary is significantly more luminous than an MS star would be at its present mass of $\sim 7 \mathrm{M}_{\odot}$. Although our model is $\sim 10 \%$ less massive than that of Andersen et al. the parameters are essentially similar.

We support this view from the results of the present paper in two ways. Firstly, the photometric fittings of Section 1 show the system to be consistent with a secondary star that is near to contact with its surrounding Roche lobe, i.e. an Algol configuration. It is difficult to establish a clear picture for the original arrangement of this massive system, but an interaction that preserves the angular momentum of the orbit would require the star centres to be implausibly near to each other at around 1 solar radius when at their closest. The generally adopted scenario thus implies that matter shed by the loser is mostly driven out of the system by intense radiation pressure, taking with it a fair proportion of the original systemic angular momentum. If about half the system's angular momentum was lost in this way, an original pair of near equal masses totalling around $30 \mathrm{M}_{\odot}$ would have had a separation of order 10 solar radii, the two near-contact components having mean radii of about 0.4 of this.

The Padova modelling (Marigo et al., 2017) giving rise to Fig 10 shows that a ZAMS star of $15 \mathrm{M}_{\odot}$ would be expanding at a rate of $\sim 0.5$ solar radii per Myr; in other words, not many Myr would elapse before the more massive star encounters its surface of limiting stability and starts to lose matter to its surroundings.

Independent confirmation of this scenario comes from Fig 6, where the position of V Pup's primary star in the luminosity-temperature diagram places it at about $1 / 3$ of the way across its Main Sequence track and just entering the instability strip corresponding to massive early type stars that are associated with the $\beta$ Cep pulsational effect. This primary star, then, appears to be behaving much as a single Population I star would behave at its deduced age and mass. We believe that this is the fourth $\beta$ cepheid variable to be reported having a precise mass measurement after V453 Cyg A (Southworth et al., 2020), one component of CW Cep (Lee \& Hong, 2010), and VV Ori (Southworth, 2021). A preliminary exploration of the pulsation spectrum was carried out, giving support to the likely nature of the low-amplitude variability, but it is likely that the power spectrum is affected by components associated with submultiples of the close binary period. This subject is rather outside the scope of the present article, but further study of the bright object V Pup is clearly called for in this connection.

The ongoing mass-loss of the secondary does raise the issue of detectable period variation, and we have confirmed the general trend of period increase reported by Qian et al. (2008), though with about a $15 \%$ lower rate. We could not confirm the $5.47 \mathrm{yr}$ additional cyclical variation found by Qian et al. after admitting more new times of minima (ToMs), though there remain relatively large discrepancies between individual ToMs and the longer term parabolic trend, particularly in the ToMs reported by Skarka (Hon̆ková et al. 2013). It is possible that some periodic term in the calculated times of minima may reduce these discrepancies, but we could not establish this with a high degree of confidence.

In this connection we note that we found an improvement in the quality of fit to the TESS data could be achieved by the inclusion of a small amount $(\sim 10 \%)$ of third light. The point can be readily ascertained by a direct comparison of the eclipse amplitudes in Figs 1 and 2; the longer-wavelength window of TESS allowing a greater proportion of light from a somewhat cooler companion to diminish the relative proportions of both eclipses. Eaton (1978) also found a third light contribution, of order $\sim 3 \%$ in the $\mathrm{V}$ range. A mid-to-late $\mathrm{B}$ type MS third star would meet the implied relative luminosity. We could not find any clear evidence of this star in the spectral data, however; though this is unsurprising given the nature of the early type rapidly rotating stars involved and their relative light levels in the optical range. The question of a third major body in the V Puppis system thus appears still unresolved and awaits further more detailed investigation.

\section{ACKNOWLEDGMENTS}

It is a pleasure to express our appreciation of the high-quality and ready availability, via the Mikulski Archive for Space Telescopes (MAST), of data collected by the TESS mission. Funding for the TESS mission is provided by the NASA Science Mission Directorate

Generous allocations of time on the $1 \mathrm{~m}$ McLellan Telescope and HERCULES spectrograph at the University of Canterbury Mt John Observatory in support of the Southern Binaries Programme have been made available through its TAC and supported by its Director, Dr. K. Pollard and previous Director, Prof. J.B. Hearnshaw. Useful help at the telescope was provided by the UCMJO management (N. Frost) assisted by F. Gunn. Considerable assistance with our use of the HRSP software package was given by its author Dr. J. Skuljan.

Encouragement and support for this programme has been shown by the the School of Chemical and Physical Sciences of the Victoria University of Wellington, as well as the Royal Astronomical Society of New Zealand and its Variable Stars South section (http://www.variablestarssouth.org). Further details on the photometry supporting the Southern Binaries Programme of the VSS are available from its Director Mark Blackford.

\section{DATA AVAILABILITY}

All data included in this article are available as listed in the paper or from the online supplementary material it refers to.

\section{REFERENCES}

Andersen, J., Clausen, J.V., Gimenez, A., Nordstrom, B., 1983, A\&A, 128, 17

Awadalla, N. S., Budding, E., 1982 Binary and multiple stars as tracers of stellar evolution: Proc. 69 IAU Coll., (Reidel), 1982, p. 239 
Bahcall, J. N., Charles, P. A., Davison, P. J. N., Stanford, P. W., Kellogg, E., York, D., 1975, MNRAS, 171, 41

Banks, T., Budding, E., 1990, ApSS, 167, 221

Bell, S. A., Adamson, A. J., Hilditch, R. W., 1987a, MNRAS, 224, 649

Bell, S. A., Kilkenny, D., Malcolm, G. J., 1987b, MNRAS, 226, 879

Blackford, M. G., Erdem, A., Sürgit, D., Özkardeş, B., Budding, E., Butland, R., Demircan, O., 2019, MNRAS, 487, 161

Budding E., 2008, APRIM 2008: Proceedings of the 10th AsianPacific Regional IAU Meeting Kunming, China, August 3-6, 2008, Eds. Shuang Nan Zhang, Yan Li, Qing Juan Yu, National Observatories of China Press, 33

Budding E., Demircan O., 2007, An Introduction to Astronomical Photometry, Cambridge Univ. Press

Budding, E., Butland, R., Blackford, M. G., 2015, MNRAS, 448, 3784

Cardelli, J. A., Clayton, G. C., Mathis, J. S., 1989, ApJ, 345, 245

Cester, B.; Fedel, B.; Giuricin, G.; Mardirossian, F.; Pucillo, M. 1977, A\&A, 61, 275

Cherepashchuk, A. M., 1976, SvAL, 2, 138

Chlebowski, T., Garmany, C. D., 1991, ApJ, 368, 241

Corcoran, M. F., 1996, MxAC, 5, 54

Cousins, A. W. J., 1947, MNAS South Africa, 6, 91

Cox, A. N., 2000, Allen's Astrophysical Quantities, p393.

Desnoux, V., Buil, C., 2005, Soc. Astron. Sci. Ann. Symp., 24, 129

Ducati, J. R., 2002, CDS/ADC Collection of Electronic Catalogues, 2237, 0

Eaton, J. A., 1978, Acta Astron., 28, 63

ESA, 1997, The HIPPARCOS and Tycho Catalogues, ESA SP1200

Friebos, H. O., 1962, Ap.J., 135, 762

Garcia, M. R., Bailyn, C. D., Grindlay, J. E., Molnar, L. A., 1989, ApJ, 341, L75

Giacconi, R., Murray, S., Gursky, H., Kellogg, E., Schreier, E., Matilsky, T., Koch, D., Tananbaum, H., 1974, ApJS, 27, 37

Gies, D. R., Bolton, C. T., 1984, ApJ, 27, 37

Groote, D., Kaufmann, J. P., Hunger, K., 1978, A\&A, 63, L9

Gummersbach C., Kaufer A., 2014, Synthetic Spectra of B MainSequence Stars from $3000-10000 \AA$, https://www.lsw.uni-heidelberg.de/projects/hot-stars /websynspec.php

Hearnshaw J. B., Barnes S. I., Kershaw G. M., Frost N., Graham G., Ritchie R., Nankivell G. R., 2002, Exp. Astron., 13, 59

Hon̆ková, K. et al.(80 authors), 2013, OEJV, 160, 1 (BRNO Cont. No. 38)

Idaczyk R., Blackford M., Butland R., Budding E., South. Stars, 2013, 52, No. 3,16

Jaschek C., Jaschek M., 1987, The Classification of Stars CUP, Cambridge

Khopolov, P. N. et al. (11 authors), 1987, GCVS, Nauka

Koch, R. H., Bradstreet, D. H., Perry, P. M., Pfeiffer, R. J., 1981, PASP, 93, 621

Kopal Z., 1959, Close Binary Systems, Chapman \& Hall, London

Kreiner, J. M., Kim, C.-H., Nha, I.-S., 2001, An Atlas of $O-C$ Diagrams of Eclipsing Binary Stars, Cracow: Wydawnictwo Nauk., AP

Kreiner, J. M., Ziółkowski, J., 1978, Acta A, 28, 497

Lee, J. W., Hong, K, 2010, arXiv 2010.03711

Leone, F., Lanzafame, A. C., 1998, A\&A 330, 306

Lubow, S. H., Shu, F. H., 1975, Ap.J, 198, 383

Maccarone, T. J., Fender, R. P., Knigge, C., Tziomis, A. K., 2009, MNRAS, 393, 1070

Mann, A. W., von Braun, K., 2015, PASP, 127, 102

Marigo P., Girardi L., Bressan A., Groenewegen M. A. T., Silva L., 2017, A\&A, 482, 833

Maury, A., 1920, HA, 84, 142

Murad, I. M.; Budding, E.1984, Observatory, 104, 83

Nowak, M. A., Heinz, S., Begelman, M. C., 2002, Ap.J., 573, 778
Pamyatnykh, A. A., 2007, CoAst, 150, 207

Parkin, E. R., Pittard, J. M., Corcoran, M. F., Hamaguchi, K., Stevens, I. R., Gosset, E., Rauw, G., De Becker, M., 2011, Bull. Soc. Roy. Sci. Liège, vol. 80, p. 610; eds. G. Rauw, M. De Becker, Y. Nazé, J-M. Vreux, P. Williams

Pittard, J. M., 2009, MNRAS, 396, 1743

Pollock, A. M. T., 1987, ApJ., 320, 283

Popowicz, A. et al. (18 authors), 2017, A\&A, 605, 26

Popper, D. M., 1947, Obs, 67, 227

Popper, D. M., 1980, Ann. Rev. A\&A, 18, 115

Priedhorshy, W. C., Terrell, J., 1983, ApJ, 273, 709

Prilutski, O. F., Usov, V. V, 1976, SvA, 20, 2

Qian, S.-B., Liao, W.-P., Fernandez-Lajus, E., 2008, Ap.J., 687, 466

Rauw, G., Nazé, Y., 2016, AdSpR, 58, 761

Ricker, G. R., et al (58 authors), 2015, JATIS, 1, id. 014003

Ruf, T., 1999, Biol. Rhythm Research, 30, 178

Schneider, D. P., Darland, J. J., Leung, K-C., 1979, AJ, 84, 236

Skuljan J., 2004, HRSP Version 2.3, Univ. Canterbury publ., p16

Skuljan J., 2012, HRSP Version 5, private communication

Smart, W. M., Textbook on Spherical Astronomy, 1960, CUP, 4th ed.

Southworth, J., Bowman, D. M., Tkachenko, A., Pavlovski, K., 2020, MNRAS 497, L19

Southworth, J., Bowman, D. M., Pavlovski, K., 2021, MNRAS $501 \mathrm{~L} 65$

Stevens, I. R., Blondin, J. M., Pollock, A. M. T., 1992, ApJ., 386, 265

Stickland, D. J., Lloyd, C., Pachoulakis, I., Koch, R. H., 1998, Obs., 118, 356

Sybesma, C. H. B., 1986, A\&A, 159, 108

Terrell, D., Munari, U., Zwitter, T., Wolf, G., 2005, MNRAS, 360, 58

Thizy, O., 2007, Soc. Astron. Sci. Ann. Symp., 26, 31

Worley, C. E., Douglass, G. G., 1997, A\&A Suppl. Ser., 125, 523)

Williams, A. S., 1886, MNRAS, 47, 91

Yakut, K., Eggleton, P. P., 2005, Ap.J., 629, 1055

York, D. G., Flannery, B., Bahcall, J., 1976, ApJ, 210, 143

Zahn J-P., 1977, A\&A, 57, 383

Zdziarski, A. A., Gierlinski, M., Wen, L., Kostrzewa, Z., 2007, MNRAS, 377, 1017 